\title{
Norois
}

Environnement, aménagement, société

206 | 2008/1

Itinéraires de lieux touristiques littoraux

\section{Itinéraires de lieux touristiques du littoral atlantique}

Routes of touristic places along the Atlantic coast

Jean-René Morice, Hélène Désiré-Pébarthe et Philippe Violier

\section{(2) OpenEdition}

1 Journals

Édition électronique

URL : https://journals.openedition.org/norois/228

DOI : 10.4000 /norois.228

ISBN : 978-2-7535-1554-3

ISSN : $1760-8546$

Éditeur

Presses universitaires de Rennes

Édition imprimée

Date de publication : 1 mars 2008

Pagination : $9-20$

ISBN : 978-2-7535-0690-9

ISSN : 0029-182X

Référence électronique

Jean-René Morice, Hélène Désiré-Pébarthe et Philippe Violier, «Itinéraires de lieux touristiques du littoral atlantique », Norois [En ligne], 206 | 2008/1, mis en ligne le 01 mars 2010, consulté le 14 janvier 2022. URL : http://journals.openedition.org/norois/228 ; DOl : https://doi.org/10.4000/norois.228

(c) Tous droits réservés 


\title{
ITINÉRAIRES DE LIEUX TOURISTIQUES DU LITTORAL ATLANTIQUE
}

\author{
Jean-René Morice, Hélène Désiré-Pébarthe et Philippe Violier \\ ESO CARTA ET LEBIM, EsThuA \\ (Université d'Angers), \\ 7 ALLÉE FranÇOIS-MitTERrand, BP 40455 - 49004 Angers \\ Jean-rene.morice@univ-angers.fr,Helene.pebarthe@univ-angers.fr,Philippe.violier@univ-angers.fr
}

\section{RÉSUMÉ}

Soit le tourisme crée des lieux urbains, soit il investit des lieux urbains existants et contribue à leur développement. Nous nous proposons dans cet article de mettre en évidence des itinéraires de lieux et ce faisant de montrer que des lieux créés par et pour le tourisme peuvent se diversifier, et que, dans des lieux investis, les acteurs s'approprient ou non le tourisme afin de développer les lieux. Enfin le tourisme peut constituer un moment de l'histoire des lieux et la fonction peut s'étioler pour peu que les conditions ne soient plus réunies.

Trois lieux touristiques de la côte atlantique sont analysés afin de rendre compte de ces itinéraires : deux, Saint-Jean-de-Monts et La Baule-Escoublac sont des stations constituées à partir du XIX siècle, le troisième une petite ville investie par le tourisme à partir des années 1820 : Les Sables-d'Olonne.

Ces trois lieux sont arrivés à une bifurcation dans leur itinéraire et évoluent différemment. L'article appréhende les raisons des divergences constatées.

Mots CLÉS : Itinéraire d'un lieu - La Baule-Escoublac - Les Sables-d'Olonne - Lieux touristiques - Saint-Jean-de-Monts - Station - Ville touristique.

\section{ABSTRACT}

\section{Routes of touristic places along the Atlantic coast}

Tourism creates urban places or invests already existing urban places and contributes to their development. The purpose of this paper is to bring to light "routes of places". It aims at showing that places created by and for tourism can diversify, and that, in places invested by tourism, the planning actors choose to appropriate or not tourism in order to increase developpement. Finally, tourism can be seen as a moment in the history of places and this activity can decrease if conditions are not put together. Three touristic places of the Atlantic coast of France are analyzed to study these routes: two of them, Saint-Jean-de-Monts and La Baule-Escoublac are seaside resorts created during the $19^{\text {th }}$ Century. The third one is a small town invested by tourism since about 1820: Les Sables-d'Olonne. These three tourists places change their routes in different ways. This paper examines how and why they have had different evolutions.

KEY WORDS : La Baule-Escoublac - Resort - Route of a place - Les Sables-d'Olonne - Saint-Jean-de-Monts - Tourist places - Tourist town. 
Les géographes qui analysent le tourisme se sont souvent essayés à l'histoire afin de retracer l'épopée qui conduisit à la création des lieux touristiques. On repère des démarches historiques chez tous les précurseurs de la géographie du tourisme. Récemment, à la fois dans le droit fil de cette réflexion, puisqu'il s'agissait de penser ensemble le temps et l'espace, et à la fois en rupture, puisque la posture réhabilite le touriste comme acteur, l'équipe Mit (2005) a dans l'ouvrage Tourismes 2, Moments de Lieux proposé un concept, le moment de lieu, pour analyser cette histoire des lieux.

Il s'agit ici d'avancer de manière complémentaire dans une autre direction et de proposer à propos de la côte atlantique, non pas des moments, donc des ruptures, mais d'appréhender l'évolution des lieux dans leur continuité, c'est le concept d'itinéraire de lieu. Si l'on admet que le tourisme est un phénomène historique, apparu de manière concomitante avec les révolutions industrielle et politique qui affectent l'Europe aux XVIII ${ }^{\mathrm{e}}$ et XIX ${ }^{\mathrm{e}}$ siècles, alors il est pertinent de s'interroger sur l'évolution et le devenir du tourisme. Il est admis que la révolution industrielle a créé pour une part, dynamisé pour une autre, des villes et que les premières peinent à passer le temps et à se reproduire au-delà du déclin de l'activité génitrice. Il apparaît peu en revanche dans la littérature géographique que le tourisme ait créé ou dynamisé des villes. Les ouvrages de géographie urbaine sont assez discrets sur ces questions comme l'a rappelé un récent colloque (« Mondes Urbains du tourisme », Duhamel et Knafou, 2007). Dès lors, il y est encore moins question de pérennité de ces lieux. Or, les lieux touristiques traversent bien le temps, et, pour certains, se font villes.

On commencera donc par montrer l'évolution divergente des lieux du tourisme, le long de la côte atlantique. Trois terrains ont été privilégiés. Les Sables-d’Olonne, Saint-Jean-de-Monts et La Baule présentent des situations très contrastées. Ensuite, on dégagera les processus qui rendent compte des divergences constatées.

\section{Le tourisme le long de la côte atlantique française}

La côte atlantique française, au sens strict, a connu des périodes fastes mais elle n’a, dans son ensemble, que ponctuellement, dans le temps et l'espace, atteint les faveurs des touristes au même niveau de fréquentation que la côte normande, côte de la Manche mer bordière, pendant les pratiques des bains froids, ou que la Côte d'Azur, depuis la généralisation des bains chauds. Pourtant le tourisme y est à peine plus récent.

\section{UnE MiSE EN TOURISME ANCIENNE SELON DEUX MODES PRINCIPAUX}

Le tourisme a commencé à apparaître le long de la côte française de l'Atlantique dès la fin du $\mathrm{XVIII}^{\mathrm{e}}$ siècle. Un premier établissement de bain est implanté à Dieppe en 1778 et deux ans plus tard on se préoccupe d'installer, pour les étrangers, des cabines le long de la plage de La Rochelle (Chadefaux, 1988). Les périodes révolutionnaire et napoléonienne reportent aux années 1820 la transformation des lieux par le tourisme et l'apparition des premiers hôtels. À notre connaissance, c'est en 1823, qu'est construit, le long de cette côte, à Arcachon, le premier hôtel à destination des touristes (Cassou-Mounat, 1977). Dans les villes anciennes, comme Dieppe et La Rochelle, les établissements destinés aux voyageurs ont suffi dans un premier temps.

Le tourisme s'est donc implanté au bord de l'Atlantique selon deux modes. Les villes anciennes en situation littorale ont d'abord été modestement investies et les transformations ont sans doute été minimes dans un premier temps. Ces lieux étaient situés sur la mer au débouché d'axes tracés à partir des grandes villes régionales ou de Paris. Le second mode est apparu plus tardivement sous la forme de lieux créés par et pour le tourisme. Arcachon semble en avoir été l'exemple le plus ancien, si l'on prend comme critère l'installation d'un premier hôtel en 1823 par un Bordelais, venant suppléer les cabanes des pêcheurs dont, jusqu'à cette date, les premiers touristes devaient se contenter (Cassou-Mounat, 1977). 
Les lieux anciens investis ou les premiers lieux créés par et pour le tourisme sont donc apparus à la fin du XVIII e siècle pour les uns et au début du XIX siècle pour les autres. Le passage, durant la seconde moitié du XIX ${ }^{\mathrm{e}}$ siècle, d'un tourisme aristocratique, réservé à une élite étroite, au tourisme en nombre, marqué par l'accès au tourisme des salariés aisés dans un contexte d'amélioration du niveau de vie, s'est traduit par deux évolutions spatiales majeures.

Premièrement, les lieux anciennement touristiques ont connu une forte expansion. Et notamment, sous la houlette d'investisseurs avisés, certains sont passés au stade industriel, caractérisé par des lotissements de plusieurs centaines de villas, lorsqu'avant 1850 on se contentait de construire par quelques unités. Arcachon (Équipe Mit, 2003) ou La Baule illustrent cette mutation. Le concept de station devrait être réservé à cette forme d'insertion du tourisme dans un espace peu ou pas bâti, à un stade industriel. Cette expansion affecte également les villes historiques comme les Sables-d'Olonne où se constitue un quartier balnéaire qui jouxte la ville ancienne (port naturel abrité par une étendue de terre à l'ouest), et la prolonge vers le sud le long de la côte. La mise en tourisme s'est faite autour de l'activité portuaire préexistante. Les quartiers de la Chaume et du centre-ville historique étaient en place dès le XV ${ }^{\mathrm{e}}$ siècle et au XVIII ${ }^{\mathrm{e}}$ siècle, les Sables-d'Olonne accueillaient déjà, outre le port de pêche, un port de commerce et des chantiers navals de renom. Les marais salants de l'arrière-pays et la vigne avaient également permis à la ville d'acquérir une certaine notoriété avant le tourisme. Les équipements liés à ce dernier s’installent d'abord à proximité des ports puis gagnent le sud le long du remblai, à l'origine mur de protection contre les assauts de la mer, qui devient en deux temps, en 1833 puis en 1866, une véritable promenade. Cette année est aussi, aux Sables, celle de l'arrivée du train qui va permettre le passage du tourisme aristocratique au tourisme en nombre.

Ensuite, un phénomène de diffusion par saut va s'opérer. Des stations nouvelles apparaissent dans les interstices entre les premières nées. La dialectique entre la pression touristique et l'inertie foncière, qu'elle émane de l'État ou des propriétaires individuels, joue périodiquement. Les élus de Saint-Jean-de-Monts durent composer avec l'État pour ouvrir l'avenue de la Mer à travers la propriété domaniale et relier le bourg à la plage. On repère des résistances mais on peut aussi observer que les grands opérateurs capitalistes se sont manifestés de manière inégale le long de la côte Atlantique. Certains secteurs, et notamment en Vendée, demeurent pour l'essentiel sous l'emprise du capital régional.

Le long des côtes de la Manche aussi bien qu'en Vendée et dans les Landes, de nouvelles stations sont donc créées sans que, en dehors de quelques secteurs bien circonscrits, comme la baie de la Baule, l'on atteigne une continuité. La façade touristique atlantique est caractérisée par ses discontinuités. Le mur de béton tant décrié reste ponctuel. Il faut aller en Belgique, au bord de la Mer du Nord, pour rencontrer une réelle façade continue.

\section{LES MOMENTS DES LIEUX}

À la différence des moments de lieu, « durée plus ou moins précise où un lieu donné (mais aussi une aire ou un réseau de lieu) incarne une situation de portée générale, dépassant l'enjeu du lieu lui-même... donc, le moment où le lieu a constitué et constitue une référence, voire un modèle pour d'autres lieux » (Équipe Mit, 2005, p. 341) les moments du lieu correspondent aux ruptures ou accélérations marquées dans l'évolution d'un lieu. Saint-Tropez, par exemple, est le lieu emblématique de l'avènement de l'élite médiatique comme catégorie dominante et créatrice de lieux, rôle exercé auparavant dans le tourisme par les élites aristocratiques et bourgeoises. Par définition, les moments de lieu sont peu nombreux. En revanche si un lieu touristique ne relève pas nécessairement d'un moment de lieu, tous les lieux touristiques ont été affectés par des moments du lieu, ne serait-ce que par le passage de l'état antérieur à l'état touristique, défini comme le moment où dans un lieu donné le tourisme est constitué en facteur marquant de l'organisation du lieu. Quels sont donc pour nos lieux laboratoires les moments du lieu? 
Le plus ancien est certainement Les Sables-d'Olonne puisque la présence d'étrangers, se baignant, est attestée dès 1816 et que la municipalité intervient afin d'éloigner les gêneurs. Si la mise en tourisme des Sables-d'Olonne est précoce, le développement touristique connaît d'abord deux phases, un développement assez lent durant une cinquantaine d'années puis une fréquentation accrue et des transformations plus nettes entre l'arrivée des premiers trains en 1866 et la Première Guerre Mondiale.

Suite à l'apparition des bains de mer est créé, en 1826, un premier établissement de bains. Le remblai est prolongé en 1833 jusqu’à la jetée des Sables. En 1846, le premier casino ouvre, créé sur une initiative privée : F. Villemaine avait acheté le terrain communal en 1841 et le projet est terminé par J. Lafeuille. Puis les premiers hôtels sont créés.

Le tourisme balnéaire va se développer fortement avec la multiplication des liaisons ferroviaires. La Compagnie des chemins de fer de Vendée est un acteur essentiel des mutations. En 1866, la gare est ouverte et les premiers trains Nantes - Les Sables commencent à circuler. En 1868, quatre allers-retours quotidiens sont assurés avec la Roche-sur-Yon à bord des « trains de plaisir ». Puis à partir de 1886, une ligne directe relie les Sables à Paris, ce qui en fait une des premières stations reliées à la capitale par voie ferrée (Huetz de Lemps, 1962).

L'essor du tourisme balnéaire des années 1870-1880 lié au train marque le réel début du processus d'aménagement touristique de la station. Si la ville encourage le développement touristique, c'est la Compagnie des chemins de fer qui a impulsé la construction de la gare puis celle du deuxième casino dans la partie ouest de la ville, à l'entrée du chenal, en 1872. Ce casino ouvre en 1876. Un nouveau quartier se développe autour de la gare, sur des marais asséchés. Le premier casino (de 1846) est détruit et remplacé par le Grand Hôtel. Cette période connaît une hausse quantitative et qualitative de l'offre d'hébergement et en 1880, on dénombre aux Sables-d'Olonne trente-cinq hôtels et environ 1000 chambres, capacité considérable pour une station à l'époque.

Un troisième casino, le Casino des Pins, dans le sud de la ville, est créé à l'initiative de J. Nicot. Il se situe dans la forêt de la Rudelière et est inauguré en 1896. Cet équipement va renforcer le poids touristique de la partie sud. L'année suivante, en 1898, un tramway, créé à l'initiative du Grand Casino, relie ce dernier, via le remblai (photo 1), au Casino des Pins et à la gare. L'intervention municipale devient alors importante. Il s'agit d'aménager la ville existante d'une part, et la rue du Buisson est ouverte, ce qui permet de créer une liaison directe entre le port et la plage. D'autre part, l'urbanisation, qui avait d'abord gagné le quartier de la gare, va gagner, notamment sous forme de spacieuses villas, la zone de la Rudelière, accessible par la corniche. Pour poursuivre cette extension vers le sud-est, la ville acquiert, en 1910, le domaine public du bois de la Rudelière, ce qui va permettre de développer, après la Première Guerre mondiale, un grand lotissement balnéaire.

Durant les années 1950, la construction d'immeubles collectifs est privilégiée, même si celle de villas se poursuit. La municipalité entreprend également en 1955 des travaux d'élargissement des rues et du remblai face à la hausse de la fréquentation et à la densification de la circulation automobile. Enfin, les aménagements touristiques sont complétés par la création d'un port de plaisance en 1975 sur des terrains gagnés sur les marais qui bordaient le fond du port : il s'agit du Port Olona, point de départ de la célèbre course du Vendée Globe.

Ainsi, en l'espace d'un siècle, le marais qui cernait la ville est repoussé à environ un kilomètre au nord de la vieille ville. Cependant, de 1900 à aujourd'hui, peu de nouveaux quartiers sont créés : la ville s'est développée par densification des quartiers existants et les Sables-d'Olonne forment aujourd'hui avec le Château-d'Olonne et Olonne-sur-Mer un ensemble urbain continu.

Depuis 2004, la municipalité affirme sa volonté de définir une stratégie de développement urbain et touristique à l'horizon 2010, stratégie dont les actions ne sont pas encore visibles mais qui constitue un nouveau jalon de l'itinéraire touristique du lieu.

En ce qui concerne La Baule, on retient le plus souvent la fondation de la gare et l'arrivée du chemin de fer en 1879, comme les actes fondateurs de la station et les complices Darlu et Hen- 


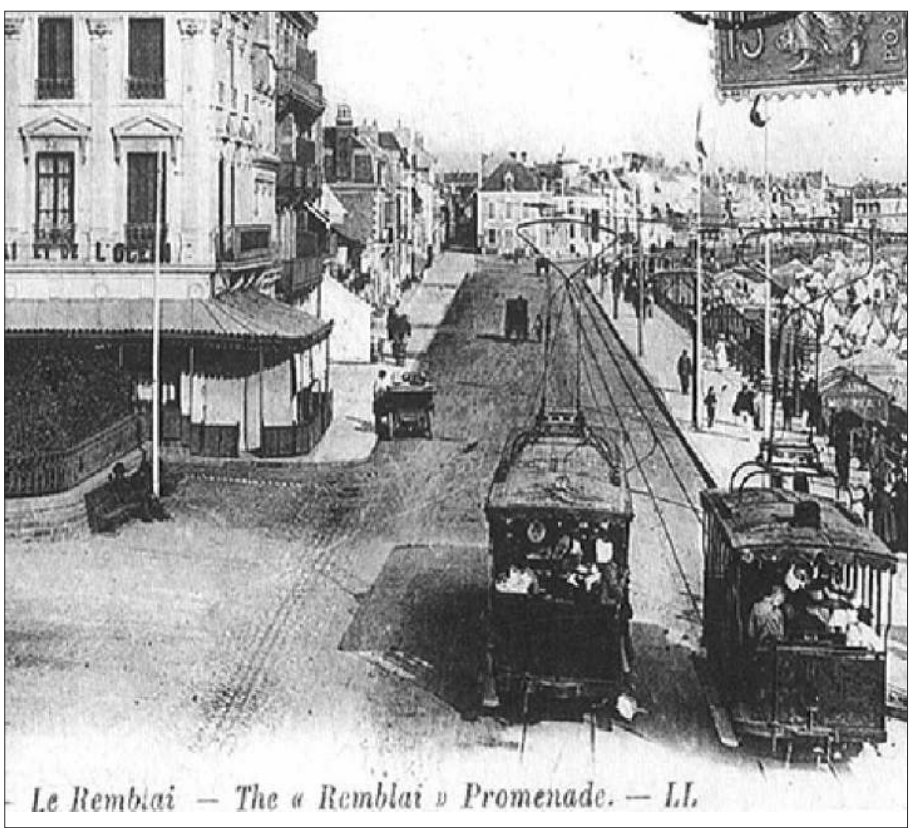

Photo 1 : Le remblai des Sablesd'Olonne, artère devenue essentielle à la ville sous l'effet du développement touristique, est doté d'un tramway en 1898

The "Remblai" promenade of Les Sables-d'Olonne became an essential street in the city under the influence of the tourist development, and was endowed with a tramway in 1898

nequart, respectivement courtier en bourse et ingénieur des chemins de fer, comme les auteurs du lieu. Si cet événement a constitué sans doute un moment du lieu, avec la projection du plan en damier de part et d'autre de l'actuelle avenue du général de Gaulle, il ne peut être retenu comme fondateur du tourisme le long de cette baie. Jean-Bernard Vighetti (2003) démontre bien que des villas commencent à être construites le long de l'actuelle plage Benoît, en prolongement du Pouliguen déjà fréquenté, dès 1864 (voir également Violier, 2002). En effet le tourisme était apparu plus tôt au Croisic. Dès 1837, la présence d'un établissement de bain y est attestée. Les légitimistes prendront leurs quartiers plutôt au Pouliguen notamment après 1853, date de la découverte du lieu par le Comte d'Escrigny (Vighetti, 2003). Du rocher, les touristes passent à la plage, qui s'étend de l'autre côté de l'étier sur le territoire d'Escoublac. Jules Benoît, le maire du Pouliguen, y avait acquis à la fin des années 1820, des terrains pour y implanter une presse à poisson. Un premier moment du lieu s'inscrit donc dans le prolongement du Pouliguen. Les Darlu et Hennequart, en investisseurs avisés, ne parient pas dans le vide, puisque la baie est déjà mise en tourisme à ses deux extrémités, mais anticipent le développement du lieu.

Un troisième moment de lieu est daté des années 1895 à 1905. La même année 1895, on inaugure le Grand Hôtel et l'Institut Verneuil dédié par son promoteur M. Pavie aux soins apportés aux tuberculeux. Le lieu est donc placé devant une bifurcation possible entre la médicalisation et la prise du pouvoir par les médecins et la poursuite du développement touristique. En 1902, la transformation de l'établissement médical en Palace atteste de l'orientation prise, de même que la prolongation du remblai par le même Pavie. Ce n'est donc pas un changement d'acteur mais l'opportunisme de l'investisseur qui décide de passer de la rentabilisation de ses capitaux par le traitement de la tuberculose à la source touristique. Dans les années 1920, La croissance de La Baule connaît une accélération et la fonction touristique est confirmée. Deux acteurs réinventent La Baule. Un certain André, déjà propriétaire de palaces à Deauville visite La Baule, plage dont il a entendu parler, et décide d'y implanter palaces, casinos et boutiques de luxe. Ses héritiers créeront le groupe Lucien Barrière. Dès lors cet acteur impulse le développement de La Baule. Le Maire Lajarrige, député de la Seine, initié à la station par son oncle, M. André, crée le lotissement de La Baule-les-Pins. 
À Saint-Jean-de-Monts, les principaux moments du lieu sont constitués par l'ouverture au tourisme, puis par le passage au stade industriel, avant que la mobilisation des acteurs, intervenue à la fin des années 1980, et montée en puissance ensuite, ne relance le développement touristique.

Il a fallu attendre 1867 pour qu'une première initiative soit prise qui lance la station. En effet, la propriété domaniale s’intercale entre le village et la plage. Les édiles avisés doivent négocier pour acheter une bande de terre et réaliser la rue de la Mer. Les débuts sont modestes et le passage à l'ère industrielle attendra les années 1950. C'est à nouveau par une initiative municipale, l'aménagement du remblai, que l'impulsion est donnée. Les promoteurs suivront et l'État apportera sa caution par l'intermédiaire du ministre Buron venu inaugurer les nouveaux équipements (Fougnie, 2006).

En conclusion, ces trois exemples permettent d'illustrer l'itinéraire de lieu et de poser quelques jalons pour comprendre les configurations prises. Nous pouvons déjà poser que c'est par une rencontre entre un lieu et des acteurs, dans un contexte donné, que se constituent des moments du lieu qui sont autant de jalons dans ces itinéraires. Comme peu de travaux historiques scientifiques se penchent sur ces questions, on doit souvent se contenter d'émettre des hypothèses. Par exemple, à la Baule, pourquoi Pavie abandonne-t-il les soins contre la tuberculose au profit du tourisme? Est-ce en raison de l'opposition de la population et des touristes craignant la contagion, dont la presse se fait écho (Vighetti, 2003), ou plutôt à cause d'un revirement avisé face aux progrès de la médecine, puisque les trois avancées fondamentales dans la guérison de la tuberculose interviennent en 1865 (inoculabilité par Villemin, en 1882, identification du germe par Koch, la même année, et découverte des rayons X en 1895 par Röntgen) ? Dans les deux cas l'acteur, localisé dans un lieu, tient compte du contexte, soit les craintes éprouvées face à une calamité, soit la fin du danger, qui constitue un risque pour le capitaliste puisqu'il lui faut réorienter ses investissements. De manière plus affirmative, la station de Saint-Jean-de-Monts, après des débuts difficiles, doit beaucoup au contexte des années 1950 et 1960. L'avènement du tourisme de masse ouvre à de nombreux lieux, repérés mais demeurés confidentiels, de passer au stade industriel. En effet, l'évolution engagée au siècle précédent se poursuit, et la diffusion sociale du tourisme tend après la seconde guerre mondiale vers l'accès de la majorité de la population au tourisme. Au contraire dans des lieux plus anciens, à la notoriété assurée, des acteurs vont s’y opposer d'autant plus efficacement que le coût d'accès, au foncier notamment, est élevé, ainsi à Dinard, à Deauville... on tente de réserver le lieu à une élite. Afin d'approfondir l'analyse, nous allons, en seconde partie, identifier les facteurs clés des bifurcations et des évolutions en cours.

\section{Des évolutions divergentes}

Alors que la fin du tourisme peut être envisagée à La Baule, où manifestement, en un lieu créé par le tourisme, on envisage sereinement qu'il ne figure plus au rang de priorité absolue, à SaintJean-de-Monts au contraire, l'accueil des touristes est toujours considéré comme essentiel. Aux Sables-d'Olonne, après bien des atermoiements, le tourisme semble considéré comme un des piliers de l'économie locale, et est clairement affirmé comme tel dans la «stratégie 2010 » mise en place actuellement. Pour appréhender ces évolutions nous proposons un modèle systémique qui articule le contexte, les stratégies des acteurs et les qualités des lieux. Dans un lieu donné, caractérisé par des qualités perçues, internes mais aussi externes (positionnement relatif, etc.), des acteurs élaborent des stratégies en fonction d'un contexte perçu et réel ce qui a pour effet de provoquer une évolution.

\section{LA QUALITÉ DES LIEUX : UNE URBANITÉ PLUS OU MOINS AFFIRMÉE ET UN POSITIONNEMENT DIFFERENCIÉ}

La qualité du lieu peut être définie comme l'ensemble des conditions du lieu, vues de l'extérieur (position absolue et relative, accessibilité...) comme de l'intérieur (cadre de vie) et qui, au regard 
de valeurs et de normes en vigueur dans une société, distinguent les lieux de manière hiérarchique et relative. Deux éléments sont déterminants pour notre sujet.

Premièrement, les lieux touristiques sont des lieux urbains au sens où ils sont constitués selon des normes et valeurs urbaines. Cette réalité est souvent mal perçue parce que l'observation est fondée sur les populations permanentes, ce qui, de fait, dévalorise les lieux touristiques. Mais si l'on prend en compte d'autres références comme l'animation, les équipements, les services, les formes urbaines, force est de constater que les lieux touristiques sont des lieux urbains (Duhamel et Knafou, 2007).

Cependant cette urbanité n'est pas homogène entre les lieux touristiques. Saint-Jean-de-Monts a une urbanité plus faible que les Sables-d'Olonne. La première localité est une station créée en marge d'un petit village et qui a été conçue dans une perspective sociale d'accès de tous aux lieux touristiques. La qualité architecturale médiocre du front de mer comme le niveau des services commerciaux notamment, constituent des indicateurs d'une urbanité faible, malgré les efforts des différentes municipalités pour diversifier la fréquentation (implantation d'un golf avec construction de villas, implantation d'un établissement de thalassothérapie...) et réhabiliter le cadre urbain (aménagement du remblai, opérations de réhabilitation des immeubles). La seconde est une ville-station, elle résulte de la juxtaposition d'une station touristique à une petite ville ancienne, disposant de fonctions administratives (sous-préfecture) et portuaires. L'ambiance urbaine y est plus vivace et subit peu la saisonnalité. Néanmoins les choix opérés actuellement par la municipalité sont axés également sur des opérations de rénovation urbaine à travers un projet de réaménagement du remblai d'une part et des efforts en matière de circulation et de stationnement d'autre part. La commune semble s'orienter vers une piétonnisation, au moins partielle, du remblai, souhaitée aussi bien par les élus que les commerçants. En outre, les Sables-d'Olonne sont engagés depuis 2002 dans une procédure de ZPPAUP afin de préserver et mettre en valeur le patrimoine bâti, notamment les villas du bord de mer.

La Baule est aussi une station mais la fréquentation par la bourgeoisie a induit un niveau d'urbanité nettement supérieur qui fait que le lieu tend à devenir un lieu résidentiel pour la population aisée de la métropole Nantes-Saint-Nazaire.

Deuxièmement, le positionnement relatif, dans l'environnement régional, des lieux touristiques évoqués est également très différent. Le développement des lieux touristiques a été pour la plupart lié à des relations avec une grande ville régionale, voire une métropole, de laquelle sont venus une partie des investissements et les premiers touristes. Cette relation binaire peut être mise en évidence à propos du couple formé par Arcachon et Bordeaux (Cassou-Mounat, 1977), par Royan avec Bordeaux également (Cassou-Mounat, 1977), Le Touquet (surnommée Paris-Plage) et Paris, Deauville et Paris... mais aussi La Baule et Nantes (Vighetti, 2003). En revanche, ni Saint-Jeande-Monts, ni les Sables n'ont bénéficié d'un tel parrainage, Challans et Luçon n'étant pas à la hauteur, pas plus que La Roche-sur-Yon fondée en 1804 par Napoléon (tableau 1).

Aujourd'hui cette proximité joue dans un autre sens, elle favorise l'établissement permanent de populations actives travaillant dans ces métropoles régionales. La Baule est ainsi concernée par un processus de résidentialisation émanant de Nantes et de Saint-Nazaire, qui affecte dans une mesure moindre Les Sables et peu Saint-Jean-de-Monts.

\section{UN CONTEXTE MARQUÉ PAR UNE PLUS GRANDE MOBILITÉ ET UNE MEILLEURE ACCESSIBILITÉ DES LIEUX}

\section{Habitat polytopique}

La mobilité croissante ainsi que l'habitat polytopique (Stock, 2005) nous apparaissent comme les faits spatiaux marquants de contexte qui affectent notamment les lieux touristiques. Rappelons la proposition. La révolution industrielle s'est accompagnée d'une recomposition de l'espace, moins traumatisante en France qu'en Angleterre certes, mais qui n'en demeure pas moins marquée par l'assignation à résidence dans un lieu de travail non choisi. Le tourisme a d'abord été, dans ce 


\begin{tabular}{|l|c|c|c|c|}
\hline & $\begin{array}{c}1999 \\
\text { Agglomération }\end{array}$ & $\begin{array}{c}1999 \\
\text { Ville }\end{array}$ & $\begin{array}{c}1936 \\
\text { Ville }\end{array}$ & \begin{tabular}{c} 
Ville \\
\hline Bordeaux
\end{tabular} \\
Nantes & 734,7 & 214,6 & 256,4 & 257 \\
Saint-Nazaire & 544,9 & 270,3 & 190,5 & 133 \\
La Roche-sur-Yon & 136,9 & 65,6 & 38,6 & 35,8 \\
Challans & 51,6 & 49,3 & 15,3 & 13,6 \\
Luçon & & 16,1 & 5,6 & 5,5 \\
\hline
\end{tabular}

Tableau 1 : Population des villes proches des lieux touristiques étudiés (Milliers d'habitants) Number of inhabitants in towns closed to tourists' places (Thousands)

contexte, un mode d'habiter compensateur car fondé sur la liberté individuelle de choisir un lieu d'habiter temporaire conçu pour la recréation, finalité du tourisme par le repos, la découverte ou le jeu (Équipe Mit, 2002). Dans ce contexte la mobilité se résumait à un va-et-vient entre un lieu de résidence et de travail, et un lieu de tourisme et de recréation.

L'accroissement du temps libre, la relativisation de la valeur travail et le recul relatif de la contrainte de la distance lié aux progrès techniques acquis dans les moyens de déplacement modifient en profondeur les conditions de l'habiter et autorisent des stratégies nouvelles. Le champ des possibles s'est ouvert.

Le concept d'habitat polytopique exprime cette idée que de nombreuses combinaisons de modes d'habiter les lieux sont expérimentées aujourd'hui. Il est possible par exemple de partager son temps entre une résidence proche du lieu de travail collectif, situé dans une grande agglomération urbaine (région parisienne), une résidence située dans un lieu touristique, dans laquelle on peut effectuer aussi des temps de travail individuel (La Baule), et des résidences temporaires dans lesquelles on adopte plutôt une posture touristique, fondée sur la recréation, soit se reposer, jouer ou découvrir.

Par ailleurs, l'arbitrage qui se faisait auparavant, face au problème de la distance, habiter un lieu de travail non choisi et bouger vers un lieu touristique choisi, n’est plus aussi nécessaire. La proximité relative entre certains lieux touristiques et les agglomérations urbaines de travail s'est réduite de telle sorte qu'il est devenu possible d'habiter en permanence les lieux des vacances.

\section{Temps d'accès}

L'accessibilité d'une destination se mesure non pas en distance mais en temps de trajet. Les trois stations étudiées sont de plus en plus accessibles, bien qu'à des niveaux différents. Si la Baule est à la fois très proche et bien reliée à la métropole nantaise et subit de ce fait un processus de résidentialisation très actif, Saint-Jean-de-Monts demeure relativement isolée. Malgré les améliorations récentes apportées, le temps d'accès demeure excessif pour des mobilités fréquentes. En effet, depuis Nantes, l'axe routier est une classique route à deux voies qui ne s'élargit à une quatre voies que sur une partie du tronçon entre Challans et Machecoul, et se limite donc à la traversée du territoire de la Vendée. Les Sables-d'Olonne sont désormais reliées au réseau autoroutier national et donc l'accessibilité depuis les villes de la vallée de la Loire en a été considérablement renforcée avec des temps de parcours de moins de deux heures depuis Nantes et Angers. Le relatif éloignement des métropoles est compensé dans le cas des Sables-d'Olonne par leur caractère de petite ville aux fonctions les rendant assez autonomes. La réouverture de ligne TGV entre Paris et Les Sables en 2008 devrait permettre un renforcement et une diversification de la clientèle touristique.

Dans ce contexte de mobilité renouvelée, l'amélioration de l'accessibilité stimule des stratégies d'acteurs. 


\section{LES STRATÉGIES DES ACTEURS DES LIEUX}

Les lieux touristiques accueillent de fait de nouvelles populations. Les populations permanentes augmentent. La littoralisation de la population affecte les lieux touristiques. L'accroissement de la population temporaire se manifeste aussi à travers la croissance du nombre des résidences secondaires.

Ces mobilités induisent des stratégies d'appropriation. Les nouveaux habitants comme les résidents temporaires veulent peser sur les décisions des élus et tendent à s'opposer à des projets ou actions à finalité touristique. Les revendications en faveur de l'amélioration du cadre de vie des habitants se font jour, dont certaines s'inscrivent en opposition aux actions visant à accroître la fréquentation touristique.

Les élus se trouvent face à un dilemme. D’une part, ils peuvent être tentés par une perspective d'annualisation de la vie du lieu. D'autre part, ils peuvent vouloir aussi maintenir et renforcer le moteur touristique de l'activité qui reste essentiel pour le lieu, mais qui reste marqué par une forte saisonnalité. À Saint-Jean-de-Monts, où le nombre de résidences principales reste plus faible (tableau 2) et où la croissance des résidences secondaires est soutenue, la stratégie évolue d'une orientation nettement touristique à une option plus binaire qui intègre désormais la présence d'une population de résidents secondaires. Mais la volonté demeure nettement affichée de développer l'activité touristique. Pour preuve, l'opportunité de la mise en œuvre en novembre d'une ligne low cost entre l'Angleterre et l'aéroport de Nantes-Atlantique a été saisie par les acteurs du tourisme qui ont élaboré des solutions pour accroître l'accessibilité du lieu en proposant des navettes et la mise à disposition à la porte de la résidence touristique de voitures de location.

À la Baule au contraire il semble que le tourisme appartienne au passé, ou du moins n'apparaisse plus comme une priorité. D'un côté la ville évolue vers une fréquentation de loisir, ce qui transparaît à travers un processus d'annualisation des services autrefois dévolus au seul tourisme; de l'autre les élus promeuvent un modèle de ville résidentielle. Les dernières élections ont notamment été marquées par l'élection d'un maire résident à l'année, traduisant les aspirations de la population à moins investir dans le tourisme et plus dans l'amélioration du cadre de vie pour la population permanente. Concernant les remarques sur l'évolution de La Baule au passé de grande station qui ne tiendrait plus son rang, le maire rétorque: «On n'est plus dans les années 30, celles des villas de prestige et des résidences secondaires. La Baule n'est plus seulement aujourd'hui une station balnéaire. C'est une vraie ville de 16500 habitants qui vit à l'année [...]. La population bauloise, composée de toutes les couches sociales, a des aspirations variées. Mon souci premier est de satisfaire d'abord ceux qui vivent à l'année ou qui reviennent chaque week-end. D'où les grands aménagements comme la rénovation du centre-ville, du bourg d'Escoublac et la réalisation de maisons de quartier à Escoublac et au Guézy [...]. Nous avons un projet de modernisation du secteur de la gare avec la création d'une sortie au nord, côté Guérande, pour permettre aux

\begin{tabular}{|l|c|c|c|}
\hline \multicolumn{1}{|c|}{1999} & Résidences principales & Résidences secondaires & Logements vacants \\
\hline Les Sables-d'Olonne & $46 \%$ & $50 \%$ & $3 \%$ \\
Château d'Olonne & $67 \%$ & $30 \%$ & $3 \%$ \\
Olonne-sur-Mer & $83 \%$ & $14 \%$ & $3 \%$ \\
Saint-Jean-de-Monts & $21 \%$ & $77 \%$ & $2 \%$ \\
La Baule-Escoublac & $38 \%$ & $59,5 \%$ & $2,5 \%$ \\
Vendée & $67 \%$ & $29 \%$ & $4 \%$ \\
France & $83 \%$ & $10 \%$ & $7 \%$ \\
\hline
\end{tabular}

Tableau 2 : Une part plus ou moins importante de résidences secondaires A more or less important part of second homes 
voyageurs de ne pas repasser par le centre [...]. J'ai arrêté des manifestations (festival du film, de la danse, Les Théâtrales) qui coûtaient très cher [...]. J'ai estimé qu'il fallait d'abord rattraper le retard d'entretien des rues et d'équipements de quartiers ${ }^{1}$. »

Le cas des Sables-d'Olonne présente quelques points communs avec celui de la Baule du fait de la présence importante de résidences secondaires. Les habitants permanents se concentrent davantage dans les deux communes voisines comme le suggèrent les $67 \%$ et $83 \%$ de résidences principales à Château d'Olonne et Olonne-sur-Mer. Autre point commun, la présence d'une population âgée importante ne pousse pas la municipalité à renforcer sa politique en matière d'animation. Aux Sables-d'Olonne, la population est même, comme le montre le tableau 3, nettement vieillissante, tandis que les deux autres communes de l'agglomération accueillent davantage de jeunes actifs.

\begin{tabular}{|l|c|c|}
\hline \multicolumn{1}{|c|}{ Territoire } & En 1982 & En 1999 \\
\hline Sables-d'Olonne & $35 \%$ & $46 \%$ \\
$\begin{array}{l}\text { Communauté de communes } \\
\text { (Sables + Château d'Olonne + Olonne-sur-Mer) }\end{array}$ & $26 \%$ & $35 \%$ \\
\hline
\end{tabular}

Tableau 3 : Une population âgée et vieillissante : part des 60 ans et plus An old and ageing population : part of people of 60 years old and more

Cependant, à la différence de la municipalité de la Baule, celle des Sables-d'Olonne affiche depuis 2004 une volonté de définir une stratégie de rénovation urbaine qui donne sa place au tourisme.

C'est suite à la création en 2003, par les professionnels du tourisme de la station (hôteliers en tête), inquiets de la stagnation de leurs chiffres d'affaire et du départ du directeur de l'office de tourisme, du "Club du Tourisme ", destiné à inciter la municipalité à agir, que les élus ont pris l'initiative de définir un plan d'actions : «les Sables 2010 ». La priorité est de résoudre des difficultés de circulation et de stationnement croissantes sous le double effet du renforcement de l'excursionnisme d'une part et de la résidentialisation d'autre part. En outre, la ville est prise, au plan urbain et touristique, dans des jeux de partenariats intercommunaux. En effet, les trois communes des Sables, de Château d'Olonne et d'Olonne-sur-Mer sont à peu près équivalentes aujourd'hui en termes de population, de taxe professionnelle récoltée et de nombre de lits touristiques. La ville des Sables-d'Olonne envisage de privilégier le développement des résidences de tourisme pour créer un nombre de lits permettant de développer l'activité d'organisation de congrès. Mais c'est à Château-d'Olonne que la résidence sera ouverte. Au plan institutionnel, c'est la communauté de communes, qui regroupe six communes, qui attribue le budget de communication touristique.

Si les Sables souffrent d'une certaine saturation foncière et sont engagés dans des partenariats intercommunaux, la ville reste la locomotive touristique de son département et cherche à se positionner face aux stations concurrentes telles qu'Arcachon ou Biarritz en réaffirmant son identité maritime et ses qualités urbaines avec notamment le slogan «la ville rêvée à la mer ».

Finalement, à Saint-Jean-de-Monts, la stratégie de développement touristique est plus poussée et plus cohérente. Sous l'impulsion de la SEM Saint-Jean Activités, la plupart des professionnels du tourisme sont mobilisés autour d'un positionnement clair, l'enfant et la famille pour la saison d'été et les vacances scolaires, les « seniors » en hors saison. Après une période, jusqu'aux années 1980, vécue comme une phase de déclin, la hausse des prix du foncier, nette depuis la fin des années 1990, comme la multiplication des résidences de tourisme et la montée en gamme des terrains de camping, de plus en plus nombreux à obtenir quatre étoiles attestent du renouveau de la fréquentation. 


\section{Conclusion}

L'analyse de l'itinéraire des lieux touristiques que sont les stations balnéaires atlantiques montre bien que des moments particuliers marquent leur évolution. Qu'il s'agisse de lieux créés par le tourisme comme la Baule ou Saint-Jean-de-Monts ou de villes existantes gagnées par cette activité, comme les Sables-d'Olonne, un système se met en place regroupant des éléments essentiels communs que sont les acteurs du lieu, son accessibilité et son urbanité, le tout dans un contexte de mobilité renforcée. Ce système est évolutif et des basculements peuvent s'opérer amenant à un renforcement du tourisme, à son maintien ou à sa disparition.

Suite à son ouverture au tourisme en 1816, l'itinéraire des Sables-d'Olonne a connu deux jalons principaux : celui du passage au tourisme en nombre à partir de 1866, puis au tourisme de masse suite à la Seconde Guerre mondiale. Le premier des deux apparaît comme le plus important eu égard à la place que le tourisme a pris dès lors dans l'économie locale et le fonctionnement urbain. La municipalité des Sables-d'Olonne a éprouvé le besoin, depuis 2004, de définir plus clairement ses objectifs en matière de développement urbain et touristique et s'affirme désormais comme une ville - station voulant assumer l'apport de l'activité touristique - laquelle est source d'une urbanité plus nette via des équipements de qualité - tout en mettant en avant aussi ses autres fonctions urbaines (portuaire et administrative).

La Baule a connu un premier basculement comparable à celui des Sables, avec l'arrivée du train, mais l'implantation des palaces a offert un second moment du lieu décisif qui a propulsé le lieu aux premiers rangs, en notoriété, de la hiérarchie des stations balnéaires européennes. Toutefois, le processus de résidentialisation en cours pousse le lieu vers une sortie du tourisme assumée par la municipalité.

À Saint-Jean-de-Monts au contraire, le tourisme demeure le moteur du lieu. La plus grande distances-temps aux métropoles et l'urbanité moins affirmée du lieu, héritée du moment de lieu des années 1950 à 1970, ne laisse guère de choix à une municipalité soucieuse de développer la ville.

\section{Bibliographie}

Cassou-Mounat (M.), 1977. - La vie humaine sur le littoral des Landes de Gascogne, thèse d'État, Université de Bordeaux, 2 tomes, 906 p.

Chadefaux (M.), 1988. - Aux origines du tourisme dans les pays de l'Adour. Du mythe à l'espace, un essai de géographie historique, Pau, J\&D Éditions, 1010 p.

Duhamel (P.), Knafou (R.), 2007. - Mondes Urbains du tourisme, Belin, Mappemonde, 368 p.

Département de la Vendée, Ville des Sables-d'Olonne, AFIT - Diagnostic stratégique et urbain, avril 2005, $148 \mathrm{p}$.

Équipe Mit, 2002. - Tourismes 1. Lieux communs, Paris, Belin, 320 p.

ÉQuipe Mit, 2005. - Tourismes 2. Moments de lieux, Paris, Belin, 349 p.

FougNie (S.), 2006. - La mutation contemporaine des stations balnéaires françaises : tentatives de modélisation de la station littorale intégrée. Études des façades Atlantiques, Manche et Mer du Nord, Thèse de Doctorat de Géographie, Université de Nantes, Igarun, 676 p. (2 vol.)

Huetz de Lemps (A.), 1962. - Les Sables-d'Olonne. Étude de Géographie historique, 1 re édition 1951, 87 p., $2^{\mathrm{e}}$ édition 1962, $132 \mathrm{p}$.

Sтоск (M.), 2006. - «L'hypothèse de l'habiter poly-topique : pratiquer les lieux géographiques dans les sociétés à individus mobiles », EspacesTemps.net, Textuel, 26 février 2006, [http://espacestemps.net/document 1853.html].

Vighetti (J.-B.), 2003. - La Baule et la presqu'île guérandaise - XIXe siècle : la naissance des bains de mer, Nantes, Siloë, (T. 1), 166 p., (T. 2), p. 179-359. 
—, 2005. - La Baule et la presqu'̂̀le guérandaise - XXe siècle : le grand essor du tourisme, Nantes, Siloë, $180 \mathrm{p}$.

Violier (P.), 2002. - La Baule de la station au lieu de vie, Paris, Belin, coll. « Mappemonde», 66, 2002, p. 20-24.

—, 2003, « Les acteurs du tourisme » dans STOск (M.) (coord.), Le tourisme : acteurs, lieux et enjeux, Belin, coll. «Sup Géographie », chapitre 5, p 167 à 208.

Cet article a été reçu le le 10 juin 2007 et définitivement accepté le 26 mars 2008. 